\title{
Counting models of temporal discrimination
}

\author{
THOMAS RAMMSAYER \\ University of Göttingen, Göttingen, Germany \\ and \\ ROLF ULRICH \\ University of Tübingen, Tübingen, Germany
}

\begin{abstract}
A three-category task was employed to test counting models for temporal discrimination. Unlike former approaches, the present one is not based on Weber functions. Specifically, the proposed test does not require the implicit but, nevertheless, debatable assumption that the pulse rate of the internal clock is constant for different durations of the standard interval. Furthermore, the present approach does not necessitate specific distributional assumptions about the interpulse times. An experiment was conducted to evaluate the predictions of this generalized counting model. The results are consistent with predictions of the generalized counting model. A further analysis suggests that the pulse rate decreases as the duration of the standard interval increases.
\end{abstract}

Internal clock models based on neural counting provide a useful heuristic for explaining human performance on the temporal discrimination of brief intervals. It is not surprising, therefore, that the notion of a pacemakercounter system represents a fundamental feature of most psychophysical models of temporal discrimination introduced over the last 4 decades (e.g., Allan, Kristofferson, \& Wiens, 1971; Creelman, 1962; Gibbon, 1977; Killeen \& Fetterman, 1988; Penton-Voak, Edwards, Percival, \& Wearden, 1996; Treisman, 1963; Treisman, Faulkner, Naish, \& Brogan, 1990).

Probably, the first quantitative model for temporal discrimination based on neural counting was developed by Creelman (1962). According to his model, the internal clock mechanism is basically characterized by an accumulator that counts neural pulses occurring during the duration to be judged. The source of the neural pulses is considered to be a large number of independent elements, whose time of firing is randomly distributed. Thus, the number of pulses counted during a given time interval is the internal representation of this interval. Creelman assumed that the number $N(t)$ of pulses accumulated during a given time interval $t$ follows a Poisson process with rate $\lambda$. This process implies that the interpulse times are independent and exponentially distributed random variables with a mean and standard deviation equal to $1 / \lambda$.

Numerous studies on duration discrimination have been conducted to test the validity of the conceptually highly

This research was partially supported by grants of the Deutsche Forschungsgemeinschaft (UL 1 16/6-1 and RA 450/9-1). The authors thank Richard Schweickert, an anonymous reviewer, and especially Peter R. Killeen for helpful comments. Correspondence concerning this article should be addressed to T. Rammsayer, Institute for Psychology, University of Göttingen, Gosslerstr. 14, D-37073 Göttingen, Germany (e-mail: trammsa@uni-goettingen.de). attractive pacemaker-counter models (e.g. Abel, 1972a, 1972b; Allan et al., 1971; Divenyi \& Danner, 1977; Getty, 1975, 1976; Killeen \& Weiss, 1987; Kinchla, 1972). In these studies, the subject typically receives two successive tones of different durations, a standard interval $t_{\mathrm{s}}$ followed by a comparison interval $t_{\mathrm{c}}$. The standard interval is usually presented first and kept constant within a single session (e.g., Getty, 1975). Within a session, the comparison interval varies randomly from trial to trial over a range of preselected values.

The most common test of counting models for temporal discrimination compares the observed Weber function with the predicted one. The observed Weber function is obtained by plotting the Weber fraction (difference threshold divided by $t_{\mathrm{s}}$ ) against $t_{\mathrm{s}}$. Usually, the observed Weber function decreases initially as $t_{\mathrm{s}}$ increases from zero and attains an asymptotic value for longer standard intervals in accordance with Weber's law, or scalar timing (Getty, 1975; Gibbon, 1977; Killeen \& Weiss, 1987; Treisman, 1963). In particular, Getty (1975) has shown that the observed Weber function decreases more sharply than the predicted Weber fraction of the simple counting model, which predicts a strictly decreasing function, a finding that has been obtained by several studies (e.g. Abel, 1972b; Fetterman \& Killeen, 1992; Grondin, 1993; Henry, 1948; Treisman,1963). Thus, counting models are at variance with the classical data from temporal discrimination tasks.

The test of the Poisson counting model just described appears inadequate, because it is based on the implicit, yet questionable, assumption that the rate of pulse generation does not vary with $t_{\mathrm{s}}$ (e.g., Getty, 1975). It might be the case that an internal timing mechanism can only operate optimally over a restricted range of durations, owing to neuronal limitations (Ivry \& Hazeltine, 1992) - that is, the rate of the pulse stream might vary with the duration of the standard interval. More specifically, it seems 
quite plausible that the timing of brief durations necessitates the generation of especially short interpulse durations (Killeen, 1992; Killeen \& Fetterman, 1988). A higher pulse rate would avoid potential truncation effects and, consequently, could improve the temporal resolution of the timing mechanism (Killeen \& Weiss, 1987). Indeed, Killeen and Weiss have proposed that an optimal timing system should be flexible and, therefore, the rate at which the internal timing mechanism operates must also vary.

Another problem in testing counting models concerns the auxiliary assumption that the distribution of $N(t)$ represents a Poisson process. Such a process implies that the interpulse time is exponentially distributed (see Ross, 1983). The mode of an exponential distribution is equal to zero. This property of the exponential distribution is incompatible with the well-known fact that, in most neurons, the action potential is followed by a refractory period, during which no action potential can be generated. Thus, it seems desirable to exclude this restrictive distributional assumption from a more general test of counting models.

The present paper introduces an alternative psychophysical test for counting models that requires neither the questionable assumption of a constant pulse rate that is independent of the duration to be timed nor a specific distributional assumption about the interpulse distribution. If, under these relaxed assumptions, the predictions of this more general test are met, this would provide substantial evidence for the validity of counting models. In particular, it would support the notion of a flexible pulse rate, as suggested by Killeen and colleagues (e.g. Killeen $\&$ Fetterman, 1988). If this test fails, however, this would challenge a large class of counting models, including those that assume that the pulse rate varies with the duration of the standard interval.

One way to test counting models without needing to vary the duration of the standard interval is the ternaryresponse task (Ulrich, 1987; Woodworth \& Schlosberg, 1954). This task has been successfully applied to test models of temporal order judgments and has yielded powerful data to invalidate false models (Allan, 1975; Sternberg, Knoll, \& Mallows, 1975; Ulrich, 1987). More specifically, the psychometric functions generated by this task revealed theoretically relevant features that were nonexistent in the psychometric functions of the standard approach. For example, the psychometric functions of the ternary task were nonmonotonic, and this feature provided strong evidence for almost all existing models of temporal order judgments. Such theoretically important information, however, is usually obscured in the standard task by the randomness of guesses on trials on which the subject is not sure what the correct response should be (Jaśkowski, 1991a). Thus, the data from the ternaryresponse task led to more detailed models on the mechanisms underlying temporal order judgment (Jaśkowski, 1991b; Stelmach \& Herdman, 1991; Sternberg et al., 1975). Therefore, it is tempting to apply the ternaryresponse task to duration discrimination to provide an alternative for testing counting models.
As in the traditional discrimination task, the subject is presented with two intervals, a standard interval S of duration $t_{\mathrm{s}}$, followed by a variable comparison interval C of duration $t_{\mathrm{c}}$. The duration $t_{\mathrm{s}}$ of $\mathrm{S}$ is again kept constant across a block of trials, whereas $t_{\mathrm{c}}$ varies randomly from trial to trial. The comparison interval is shorter than, equal to, or longer than $t_{\mathrm{s}}$. In contrast to the classical task, however, the subject is required to judge whether $\mathrm{S}$ was shorter than, longer than, or equal to $\mathrm{C}$. These three response alternatives will be denoted by " $\mathrm{C}>\mathrm{S}$," " $\mathrm{S}>\mathrm{C}$," and " $\mathrm{S}=$ $\mathrm{C}$," respectively. Thus, the ternary-response task allows the estimation of probabilities $\operatorname{Pr}\left\{\right.$ “S $\left.>\mathrm{C} " \mid t_{\mathrm{s}}, t_{\mathrm{c}}\right\}$, $\operatorname{Pr}\left\{\right.$ “C $>\mathrm{S}$ ” $\left.\mid t_{\mathrm{s}}, t_{\mathrm{c}}\right\}$, and $\operatorname{Pr}\left\{\right.$ “S $\left.=\mathrm{C} " \mid t_{\mathrm{s}}, t_{\mathrm{c}}\right\}$ as a function of $\left(t_{\mathrm{s}}, t_{\mathrm{c}}\right)$. In the present paper, these estimates will be compared with the predicted probabilities of the generalized counting model. A separate test will be performed at each level of $t_{\mathrm{s}}$. Unlike former approaches, this test does not require specific distributional assumptions about the interpulse time distribution (see Appendix A for mathematical details).

The present experiment employed two standard intervals of 50 and 1,000 msec. For each standard interval there were nine comparison intervals, and subjects were asked to indicate in each trial whether the standard appeared to be shorter than, equal to, or longer than the comparison. Thus, for each standard, a family of psychometric functions was generated and compared with the predicted functions of counting models. A computer routine estimated the two parameters $\mu^{*}=\mu / \delta$ and $\sigma^{*}=\sigma^{2} / \mu$ by minimizing the root-mean squared (RMS) error between predicted and observed response probabilities. Note that $\mu$ and $\sigma$ denote the mean and the standard deviation of the interpulse interval, respectively, and $\delta$ denotes a criterion count that separates contiguous response categories. Although, $\mu^{*}$ and $\sigma^{*}$ can be identified only, these two estimates nevertheless permit crucial inferences about the underlying interpulse distribution, as we will explain later.

In sum, then, we assess the predictions of a counting model that does not make any distributional assumptions about interpulse intervals. These predictions, which are derived in Appendix A, will be employed to estimate certain parameters of the interpulse distribution from the data collected by means of a ternary-response paradigm. These parameters will be estimated for two different standard intervals, $t_{\mathrm{s}}=50 \mathrm{msec}$ and $t_{\mathrm{s}}=1,000 \mathrm{msec}$. Besides assessing the goodness of model fit, this allows us to test whether the parameter estimates of the interpulse distribution will vary with the duration of the standard interval.

\section{METHOD}

\section{Subjects}

One male and 3 female students, ranging from 21 to 34 years of age $(M=26.7$ years $)$, participated in this experiment.

\section{Stimuli}

Auditory stimuli were white-noise bursts with zero rise and fall times. All the stimuli were presented binaurally through headphones at an intensity of $67 \mathrm{~dB}$ (SPL). 


\section{Procedure}

There were two experimental conditions; in the first experimental condition the standard interval was $t_{\mathrm{s}}=50 \mathrm{msec}$, and in the second condition it was $t_{\mathrm{s}}=1,000 \mathrm{msec}$. Each daily testing session consisted of two blocks of 150 trials each, with a 1-min pause between blocks. The standard interval was constant during a single session but varied between sessions.

The first time interval presented was the standard interval $\left(t_{\mathrm{s}}\right)$. After an interstimulus interval of $900 \mathrm{msec}$, a second interval, the comparison interval $\left(t_{\mathrm{c}}\right)$, was presented. The duration of the variable comparison interval was $t_{\mathrm{c}}=t_{\mathrm{s}}+\Delta$, where the difference interval $\Delta$ is experimentally varied according to the method of constant stimuli. More specifically, let $U$ be an individually determined step size; then, the difference interval $\Delta_{i}$ can be defined as $\Delta_{i}=(i-5) \cdot U$ $(i=1, \ldots, 9)$, resulting in four comparison intervals shorter and four comparison intervals longer than the standard interval. Within each experimental condition, there were 100 trials with each possible duration of the comparison interval, except for $\Delta_{5}=0$ (i.e., $t_{\mathrm{s}}=$ $t_{\mathrm{c}}$ ). In this case, there were 400 trials, resulting in a total number of 1,200 trials for each experimental condition. Comparison intervals were presented in random order. In order to avoid ceiling and floor effects, an individual step size $U$ was determined for each subject and each experimental condition in a preceding training session. For $t_{\mathrm{s}}=50 \mathrm{msec}\left(t_{\mathrm{s}}=1,000 \mathrm{msec}\right)$, these step sizes were $2,4,5$, and $6 \mathrm{msec}$ (50, 45, 60, and $70 \mathrm{msec})$ for subjects J.M., B.O., A.M., and C.M., respectively.

Each subject was seated at a table with a keyboard and a computer monitor in a sound-attenuated room. To initiate a trial, the subject pressed the space bar; the auditory presentation began $900 \mathrm{msec}$ later. The two intervals were presented with an interstimulus interval of $900 \mathrm{msec}$. The subject's task was to indicate one of three possible decisions regarding the duration of the two intervals presented within each trial by pressing one of three designated keys on the keyboard. The response keys were labeled "first interval longer," "second interval longer," and "same duration." After each response, visual feedback ("+" [i.e., correct] or "-" [i.e., false] ) was displayed for $1 \mathrm{sec}$. One second after the feedback, the next trial started.

\section{RESULTS}

Observed psychometric functions and those predicted by counting models are shown in Figure 1. As can be seen, counting models provide an excellent fit for the observed data. As one would expect, the spread of the psychometric functions is larger for $t_{\mathrm{s}}=1,000 \mathrm{msec}$ than for $t_{\mathrm{s}}=$

Table 1

Estimated Model Parameters $\left(\mu^{*}, \sigma^{*}\right)$ and Root-Mean Square (RMS) for Each Subject and Standard Duration

\begin{tabular}{lccccc}
\hline Subject & $\mu^{*}$ & \multicolumn{1}{c}{$\sigma^{*}$} & RMS & PSE & DL \\
\hline \multicolumn{5}{c}{ Part 1 : Standard Duration $=50 \mathrm{msec}$} \\
B.O. & 4.4 & 0.1 & .02 & $50.1 \pm 0.3$ & $2.6 \pm 0.1$ \\
C.M. & 8.5 & 0.4 & .03 & $50.2 \pm 0.4$ & $4.3 \pm 0.5$ \\
A.M. & 5.7 & 0.4 & .04 & $49.2 \pm 0.5$ & $4.5 \pm 0.3$ \\
J.M. & 2.3 & 0.1 & .05 & $50.5 \pm 0.1$ & $1.4 \pm 0.1$ \\
\multicolumn{7}{c}{} \\
B.O. & 56.3 & 1.1 & .04 & $995.6 \pm 4.3$ & $34.6 \pm 2.2$ \\
C.M. & 92.4 & 5.8 & .04 & $985.5 \pm 9.1$ & $75.7 \pm 4.7$ \\
A.M. & 71.7 & 3.7 & .04 & $982.9 \pm 5.5$ & $59.0 \pm 4.7$ \\
J.M. & 45.9 & 2.0 & .06 & $979.4 \pm 3.1$ & $39.8 \pm 3.7$ \\
\hline
\end{tabular}

The table also provides the point of subjective equality (PSE; $\pm S E$ ) and the difference limen (DL; $\pm S E$ ) in columns 5 and 6 , respectively. The unit of measurement for $\mu^{*}, \sigma^{*}$, PSE, and DL is the millisecond. Standard errors (SEs) were determined by the method of bootstrapping (Mooney \& Duval, 1993).
$50 \mathrm{msec}$, because temporal discrimination is known to deteriorate as the standard interval increases. Table 1 gives the mean RMS for each subject and standard interval and also contains the estimated parameters $\mu^{*}$ and $\sigma^{*}$.1

The parameter $\mu^{*}$ establishes an upper bound for the mean interpulse time $\mu$ according to $\mu=\mu^{*} / \delta$. Because $\delta \geq 1$ must hold, $\mu$ cannot be larger than $\mu^{*}$. Appendix B contains further bounds for $\mu, \sigma$, and $\delta$ under the reasonable assumption that the coefficient of variation of the interpulse distribution is less than or equal to one. Specifically, it is shown that the upper bound of the criterion $\delta$ should be equal to the ratio $\mu^{*} / \sigma^{*}$. Furthermore, the mean $\mu$, as well as the standard deviation $\sigma$, of the interpulse distribution should be larger than $\sigma^{*}$.

For the present data, the mean estimate of $\mu^{*}$ increased significantly from $5.6 \mathrm{msec}$ for $t_{\mathrm{s}}=50 \mathrm{msec}$ to $66.6 \mathrm{msec}$ for $t_{\mathrm{s}}=1,000 \mathrm{msec}(t=6.95, d f=3, p=.003) .^{2}$ This large increase in $\mu^{*}$ could reflect an increase in the mean interpulse time $\mu$, the criterion $\delta$, or both parameters. Because $\mu$ is inversely related to the pulse rate, a decrease in $\mu$ would mean that the pulse rate decreases as $t_{\mathrm{s}}$ becomes longer.

Theoretically more important, the average estimate of $\sigma^{*}=\sigma^{2} / \mu$ was significantly larger for $t_{\mathrm{s}}=1,000 \mathrm{msec}$ than for $t_{\mathrm{s}}=50 \mathrm{msec}$; the estimates were 3.2 and 0.25 , respectively, $(t=3.03, d f=3, p=.028)$. This result clearly shows that some aspects of the interpulse distribution change with $t_{\mathrm{s}}$. Such a finding is incompatible with the implicit common notion, according to which the interpulse distribution does not vary with $t_{\mathrm{s}}$ (e.g., Getty, 1975).

A further analysis shows that the upper bound of $\delta$ is about 21 pulses; surprisingly, this conclusion applies to both standard intervals. For the short standard, $\mu$ lies somewhere between 0.3 and $5.6 \mathrm{msec}$, whereas for the long standard, $\mu$ ranges from 3.2 to $66.6 \mathrm{msec}$. The smallest possible value of $\sigma$ is 0.3 and $3.2 \mathrm{msec}$ for the short and the long standard intervals, respectively.

\section{DISCUSSION}

As was reviewed in the introduction, previous psychophysical studies on temporal discrimination suggest that the predicted Weber functions of Poisson counting models are at variance with the empirically determined functions. Specifically, the observed Weber fractions decrease more sharply than would be predicted by the Poisson counting model. Furthermore, the observed Weber function usually approaches a lower asymptote larger then zero, whereas the predicted Weber function decreases strictly toward zero. The reasons for these discrepancies may be at least threefold.

First, an implicit assumption of counting models holds that the pulse generation process is independent of the duration of the standard interval (e.g., Abel, 1972a, 1972b; Allan et al., 1971; Divenyi \& Danner, 1977; Getty, 1975, 1976; Grondin, 1993; Killeen \& Weiss, 1987; Kinchla, 1972; Treisman, 1963). It seems plausible, however, that the rate of the pulse stream changes with the duration of the standard interval (Killeen, 1992; Killeen \& Fetterman, 

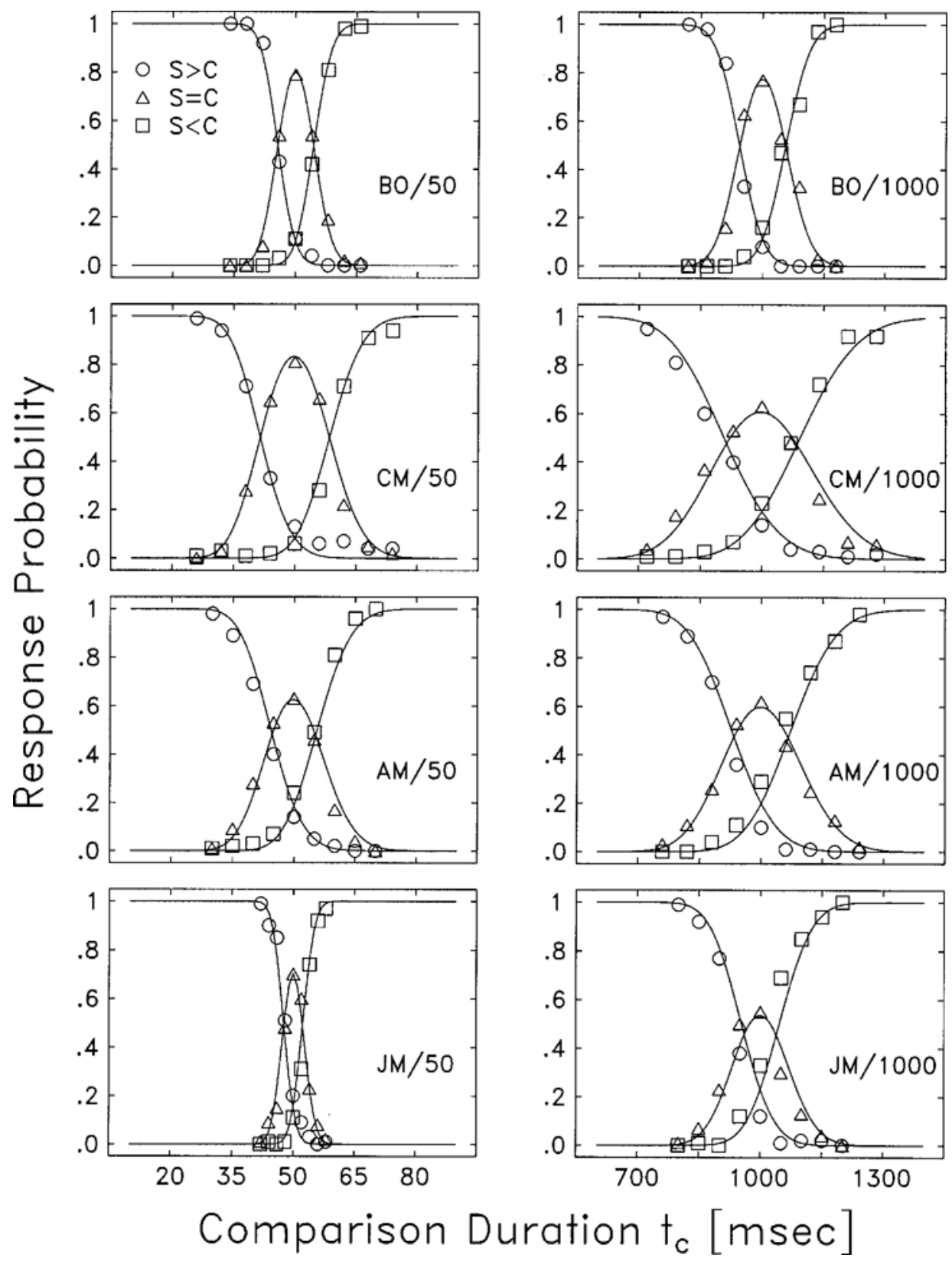

Figure 1. Observed and predicted psychometric functions for each subject and each standard duration. Left panels: standard duration $t_{\mathrm{s}}=50 \mathrm{msec}$. Right panels: $t_{\mathrm{s}}=1,000 \mathrm{msec}$. Top row: subject B.O. Second row from top: subject C.M. Third row from top: subject A.M. Bottom row: subject J.M.

1988). For example, shorter durations may necessitate a higher pulse rate to improve the precision of timing.

Second, counting models for duration discrimination assume that the pulse generation stream follows a Poisson process. Such a process implies an exponential interpulse distribution having a modal value of zero. In a more realistic counting process, however, one has to proceed from some refractory period of both the generation and the counting of pulses, which would render this distribution rather unrealistic. In addition, errors in counting would imply that the interpulse time distribution of the internal counting mechanism represents a probabilistic mixture of several interpulse distributions. Such an imperfect counting process arises when the internal clock fails to register each pulse (Killeen \& Weiss, 1987). With imperfect counting, the interpulse distribution of two successively registered pulses depends not only on the interpulse time of the pulse generation process, but also on the num- 
ber of pulses not registered. The more pulses that fail to register, the longer will be the interpulse time between two registered pulses. More specifically, if the counting process fails to register $n$ successive pulses, this interpulse time would follow a $(n+1)$-step gamma distributioninstead of an exponential distribution, as the standard Possion model implies. Because such an imperfect counting process is probabilistic (sometimes only one pulse is lost, sometimes two, etc.), the interpulse time would follow a probabilistic mixture of gamma distributions.

Finally, the traditional psychophysical approach for testing counting models forces the subject to choose between only two response categories (standard longer than comparison vs. standard shorter than comparison). The problem with this approach is that subjects cannot indicate that both intervals appear equally long, a state that will occur when an equal number of counts is accumulated during each interval. This restriction may bias the evaluation of the counting process, since the occurrence of this state has not been taken into account in the derivation of the predicted Weber function (see Getty, 1975). Since the ternary-response approach includes an additional response category for same judgments, subjects were no longer required to select an inappropriate category when the two intervals were perceived as equally long (see Jaśkowski, 1991a).

The results of the present experiment are clearly consistent with the predictions of counting models. The predicted and observed psychometric functions agreed surprisingly well, despite the fact that only two parameters $\left(\sigma^{*}, \mu^{*}\right)$ had to be estimated for each data set. It is important to note that a good fit might not be persuasive, especially when the results would not be a surprise to anyone (Roberts \& Pashler, 2000). Given the background of previous studies on temporal order judgments also employing this ternary-response task (Jaśkowski, 1991b; Ulrich, 1987), the good fit observed in the present study was quite unexpected. The psychometric functions assessed in these former studies were often nonmonotonic. In fact, many more parameters than two were necessary to provide an acceptable model fit (Jaśkowski, 1991b).

Even more important, however, was another outcome of the present study. Although, the model parameters $\mu$, $\sigma$, and the criterion $\delta$ were not identifiable, the estimates of $\sigma^{*}=\sigma^{2} / \mu$ mean clearly argue against the implicit assumption that the process of pulse generation operates identically for short and long intervals. This conclusion holds for any pulse generation process, including the usually assumed Possion process.

Furthermore, these estimates of $\sigma^{*}$ indicate that the mean interpulse time increases with the duration of the standard interval if one proceeds from the additional, yet reasonable, assumption that the mean and the standard deviation are positively correlated. This assumption is highly plausible, since in almost all neural latency mechanisms, both measures are positively associated (e.g., Luce, 1986, p. 65). In addition, in most theoretical latency distribu- tions, both parameters cannot vary orthogonally but are positively related to each other (e.g. Cox, 1967; McGill, 1963). Specifically, let us assume that the standard deviation is approximately linearly related to its mean - that is, $\sigma=c \cdot \mu, c>0$. Under this condition, we have $\sigma^{*}=$ $\sigma^{2} / \mu=c^{2} \cdot \mu$. Then, by inference, the pulse rate is reduced when subjects are required to discriminate between longer intervals. This converging evidence from human subjects supports the notion deduced from animal timing that the mean interpulse time varies proportionately with the interval being timed (Killeen \& Fetterman, 1988).

The idea of a duration-dependent pulse rate might be unexpected from a psychophysical point of view. However, from a pragmatic perspective, such a change of the pulse rate may represent an optimization of the timing process. It is conceivable that the production of each pulse consumes a certain amount of limited resources and, thus, the pulse production process requires a tradeoff between precision and demand for resources. For example, although for the discrimination of very brief intervals, a relatively high pulse rate would be needed to avoid truncation, as compared with the discrimination of longer intervals, a much lower pulse rate will suffice for the accurate timing of longer intervals. Adaptation of pulse rate to the duration of the standard interval could be achieved either by a large pool of different oscillating systems or pacemakers, from which the most appropriate one can be selected (Miall, 1996), or from a mechanism to adjust the period of the pulse generation process to the base duration of the intervals to be timed (Torras, 1985; Treisman \& Brogan, 1992; Treisman et al., 1990).

As was mentioned earlier, extant data showed that the observed Weber fractions decrease more sharply than would be predicted by the fixed-rate Poisson model. Moreover, the observed Weber function usually approaches a lower asymptote larger than zero, whereas the predicted Weber function derived from the Poisson model decreases continuously toward zero. The variable-rate counting model introduced in this paper accounts for the observed constancy of the Weber function if one assumes that the mean interpulse time increases linearly with $t_{\mathrm{s}}$. Or in other words, this constancy is achieved only if the number of average counts does not vary with $t_{\mathrm{s}}$.

In summary, the present study clearly demonstrates the general validity of counting models for the temporal discrimination of brief intervals. The failure of previous psychophysical studies to validate counting models appears to be basically due to the implicit assumption of a duration-independentpulse rate. Further restrictions (e.g., the assumption of exponentially distributed interpulse times and the requirement to choose between only two response categories) could also have contributed to this failure. The present analysis suggests that the pulse rate changes with the duration of the standard interval. Further research in temporal discrimination is needed to strengthen this conclusion and to elucidate the mechanisms that are involved in the control of the pulse rate. 


\section{REFERENCES}

AвEL, S. M. (1972a). Discrimination of temporal gaps. Journal of the Acoustical Society of America, 52, 519-524.

ABEL, S. M. (1972b). Duration discrimination of noise and tone bursts. Journal of the Acoustical Society of America, 51, 1219-1223.

Allan, L. G. (1975). The relationship between judgments of successiveness and judgments of order. Perception \& Psychophysics, 18, 29-36.

Allan, L. G., Kristofferson, A. B., \& Wiens, E. W. (1971). Duration discrimination of brief light flashes. Perception \& Psychophysics, 9 , 327-334.

Cox, D. R. (1967). Renewal theory (2nd ed.). London: Methuen.

Creelman, C. D. (1962). Human discrimination of auditory duration. Journal of the Acoustical Society of America, 34, 582-593.

Divenyi, P. L., \& DANNER, W. F. (1977). Discrimination of time intervals marked by brief acoustic pulses of various intensities and spectra. Perception \& Psychophysics, 21, 125-142.

Fetterman, G., \& Killeen, P. R. (1992). Time discrimination in Columbia livia and Homo sapiens. Journal of Experimental Psychology: Animal Behavior Processes, 18, 80-94.

GETTy, D. J. (1975). Discrimination of short temporal intervals: A comparison of two models. Perception \& Psychophysics, 18, 1-8.

GetTy, D. J. (1976). Counting processes in human timing. Perception \& Psychophysics, 20, 191-197.

Gibbon, J. (1977). Scalar expectancy theory and Weber's law in animal timing. Psychological Review, 84, 279-325.

Grondin, S. (1993). Duration discrimination of empty and filled intervals marked by auditory and visual signals. Perception \& Psychophysics, 54, 383-394.

HENRY, F. M. (1948). Discrimination of the duration of a sound. Journal of Experimental Psychology, 38, 734-743.

Ivry, R. B., \& Hazeltine, R. E. (1992). Models of timing-with-a-timer. In F. Macar, V. Pouthas, \& W. J. Friedman (Eds.), Time, action and cognition: Towards bridging the gap (pp. 183-189). Dordrecht, The Netherlands: Kluwer.

JAŚKOWSKI, P. (1991a). Perceived onset simultaneity of stimuli with unequal durations. Perception, 20, 715-726.

JAŚKOWSKI, P. (1991b). Two-stage model for order discrimination. Perception \& Psychophysics, 50, 76-82.

KilleEN, P. R. (1992). Counting the minutes. In F. Macar, V. Pouthas, \& W. J. Friedman (Eds.), Time, action and cognition: Towards bridging the gap (pp. 203-214). Dordrecht, The Netherlands: Kluwer.

Killeen, P. R., \& Fetterman, J. G. (1988). A behavioral theory of timing. Psychological Review, 95, 274-295.

Killeen, P. R., \& Weiss, N. A. (1987). Optimal timing and the Weber function. Psychological Review, 94, 455-468.

KINCHLA, J. (1972). Duration discrimination of acoustically defined intervals in the 1- to 8-sec range. Perception \& Psychophysics, 12, 318-320.

LuCE, R. D. (1986). Response times: Their role in inferring elementary mental organization. New York: Oxford University Press.

McGiLL, W. J. (1963). Stochastic latency mechanisms. In R. D. Luce, R. R. Bush, \& E. Galanter (Eds.), Handbook of mathematical psychology (Vol. 1, pp. 309-360). New York: Wiley.

Miall, C. (1996). Models of neural counting. In M. A. Pastor \& J. Artieda (Eds.), Time, internal clocks and movement (pp. 69-94). Amsterdam: Elsevier.

Mooney, C. Z, \& Duval, R. D. (1993). Bootstrapping: A nonparametric approach to statistical inference. Beverly Hills, CA: Sage.

Penton-Voak, I. S., Edwards, H., Percival, A., \& Wearden, J. H.
(1996). Speeding up an internal clock in humans? Effects of click trains on subjective duration. Journal of Experimental Psychology: Animal Behavior Processes, 22, 307-320.

Roberts, S., \& PAshler, H. (2000). How persuasive is a good fit? A comment on theory testing. Psychological Review, 107, 358-367.

Ross, S. M. (1983). Stochastic processes. New York: Wiley.

Stelmach, L. B., \& Herdman, C. M. (1991). Directed attention and perception of temporal order. Journal of Experimental Psychology: Human Perception \& Performance, 17, 539-550.

Sternberg, S., Knoll, R. L., \& Mallows, C. L. (1975). Conditions for parallel psychometric functions based on rating-scale data: Applications to temporal-order judgments (Tech. memorandum). Murray Hill, NJ: Bell Laboratories.

TorRas, C. I. G. (1985). Pacemaker neuron model with plastic firing rate: Entrainment and learning ranges. Biological Cybernetics, 52, 79-91.

Treisman, M. (1963). Temporal discrimination and the indifference interval: Implications for a model of the "internal clock." Psychological Monographs, 77, 1-31.

Treisman, M., \& Brogan, D. (1992). Time perception and the internal clock: Effects of visual flicker on the temporal oscillator. European Journal of Cognitive Psychology, 4, 41-70.

Treisman, M., Faulkner, A., Naish, P. L. N., \& Brogan, D. (1990). The internal clock: Evidence for a temporal oscillator underlying time perception with some estimates of its characteristic frequency. Perception, 19, 705-743.

UlRICH, R. (1987). Threshold models of temporal-order judgments evaluated by a ternary response task. Perception \& Psychophysics, 42, 224-239.

Woodworth, R. S., \& Schlosberg, H. (1954). Experimental psychology (3rd ed.). London: Methuen.

\section{NOTES}

1. We also performed a conventional psychophysical data analysis for three-category experiments (see Woodworth \& Schlosberg, 1954). This analysis provides the point of subjective equality (PSE) and the difference limen (DL) for each subject and each standard interval (see Table 1). The PSE was virtually identical to the corresponding standard interval for $t_{\mathrm{s}}=50 \mathrm{msec}$. However, the PSE undershot slightly the standard interval for $t_{\mathrm{s}}=1,000 \mathrm{msec}$. As would be predicted by Weber's law, the absolute discrimination performance deteriorated clearly from $\mathrm{DL}=3.2$ to $52.3 \mathrm{msec}$ as $t_{\mathrm{s}}$ increased from 50 to $1,000 \mathrm{msec}(t=5.56, d f=3, p=$ $.006)$. The mean Weber fractions were .064 and .052 for $t_{\mathrm{s}}=50 \mathrm{msec}$ and $t_{\mathrm{s}}=1,000 \mathrm{msec}$, respectively, and did not differ significantly $(p=.145)$.

2. We employed the so-called bootstrapping procedure (Mooney \& Duval, 1993) to compute standard errors for the estimates of $\mu^{*}$ and $\sigma^{*}$. In essence, this procedure takes the observed response probabilities as estimates for the true response probabilities. These estimates are used to simulate the experiment again, yielding a bootstrap sample. For each bootstrap sample, the parameters $\mu^{*}$ and $\sigma^{*}$, referred to as bootstrap replications, are estimated. These replications generate the sampling distribution for each parameter. The standard deviation of this distribution provides the standard error of the corresponding parameter. For example, we generated 500 bootstrap samples for the data of subject B.O. under the $t_{\mathrm{s}}=50 \mathrm{msec}$ condition and estimated for each sample the parameters $\mu^{*}$ and $\sigma^{*}$. Thus, we obtained a standard error of 0.3 for $\mu^{*}$ and 0.02 for $\sigma^{*}$. This example shows that the estimates obtained are quite reliable. In all other cases, the reliability of the estimates was virtually identical. We thank Peter R. Killeen for suggesting this procedure. 


\section{APPENDIX A}

Predicted Response Probabilities of the Generalized Counting Model

According to a standard theorem of renewal theory, the number of pulses $N(t)$ counted during the time interval $t$ is approximately normally distributed with a mean of $t / \mu$ and a variance of $t \sigma^{2} / \mu^{3}$, where $\mu$ and $\sigma$ denote the mean and standard deviation of the interpulse duration, assumed to be finite (see, e.g., Ross, 1983, p. 62). No assumptions about the interpulse distribution are required, although as in the Poisson counting process, successive pulses are assumed to be independent.

As a simple demonstration, suppose that the interpulse distribution follows an exponential distribution with a mean of $\mu=$ $5 \mathrm{msec}$. For an exponential random variable, the standard deviation is equal to its mean-that is, $\sigma=5 \mathrm{msec}$. Assume that we are interested in computing the probability $\operatorname{Pr}\{N(40) \leq 3\}$. In this particularcase, $N(t)$ follows a Poisson distribution, and thus the exact value of this probability is

$$
\begin{aligned}
\operatorname{Pr}\{N(40) \leq 3\} & =\sum_{i=0}^{3} \exp (-40 / 5) \frac{(40 / 5)^{i}}{i !} \\
& =.038 .
\end{aligned}
$$

In contrast, the approximated value is

$$
\begin{aligned}
\operatorname{Pr}\{N(40) \leq 3\} & \approx \Phi\left[\frac{3-8}{\sqrt{8}}\right] \\
& \approx .038 .
\end{aligned}
$$

which is sufficiently close to the exact value for our purposes. (The approximation improves for longer durations-that is, when $t$ increases.) The computation of exact probabilities for a nonexponentialinterpulse distributionis possible but would generally be tedious (see, e.g., Ross, 1983, pp. 56-57).

The above standard theorem allows the calculations of the response probabilities $\operatorname{Pr}\left\{\right.$ “S $\left.>\mathrm{C} " \mid t_{\mathrm{s}}, t_{\mathrm{c}}\right\}, \operatorname{Pr}\left\{\right.$ “C $\left.>\mathrm{S} \mid t_{\mathrm{s}}, t_{\mathrm{c}}\right\}$, and $\operatorname{Pr}\left\{\right.$ “S $\left.=\mathrm{C} " \mid t_{\mathrm{s}}, t_{\mathrm{c}}\right\}$. Since $N\left(t_{\mathrm{s}}\right)$ and $N\left(t_{\mathrm{c}}\right)$ are (approximately) normally distributed, the difference $D\left(t_{\mathrm{s}}, t_{\mathrm{c}}\right)=N\left(t_{\mathrm{c}}\right)-N\left(t_{\mathrm{s}}\right)$ also follows a normal distribution. In particular, the mean of this difference is

$$
\begin{aligned}
\mathrm{E}\left[D\left(t_{\mathrm{s}}, t_{\mathrm{c}}\right)\right] & =\mathrm{E}\left[N\left(t_{\mathrm{c}}\right)-N\left(t_{\mathrm{s}}\right)\right] \\
& =\mathrm{E}\left[N\left(t_{\mathrm{c}}\right)-N\left(t_{\mathrm{s}}\right)\right] \\
& =\frac{1}{\mu}\left(t_{\mathrm{c}}-t_{\mathrm{s}}\right),
\end{aligned}
$$

with the variance

$$
\begin{aligned}
\operatorname{Var}\left[D\left(t_{\mathrm{s}}, t_{\mathrm{c}}\right)\right] & =\operatorname{Var}\left[N\left(t_{\mathrm{c}}\right)\right]+\operatorname{Var}\left[N\left(t_{\mathrm{s}}\right)\right] \\
& =\frac{t_{\mathrm{c}} \sigma^{2}}{\mu^{3}}+\frac{t_{\mathrm{s}} \sigma^{2}}{\mu^{3}} \\
& =\frac{\sigma^{2}}{\mu^{3}}\left(t_{\mathrm{c}}+t_{\mathrm{s}}\right) .
\end{aligned}
$$

With Equations 5 and 6, the probability $\operatorname{Pr}\left\{\right.$ “ $\mathrm{S}>\mathrm{C}$ " $\left.\mid t_{\mathrm{s}}, t_{\mathrm{c}}\right\}$ is computed as

$$
\begin{aligned}
\operatorname{Pr}\left\{\text { “S }>\mathrm{C} " \mid\left(t_{\mathrm{s}}, t_{\mathrm{c}}\right)\right\} & =\operatorname{Pr}\left\{N\left(t_{\mathrm{s}}\right)-N\left(t_{\mathrm{c}}\right)>\delta\right\} \\
& =\operatorname{Pr}\left\{N\left(t_{\mathrm{c}}\right)-N\left(t_{\mathrm{s}}\right) \leq-\delta\right\} \\
& =\operatorname{Pr}\left\{D\left(t_{\mathrm{s}}, t_{\mathrm{c}}\right) \leq-\delta\right\} \\
& =\Phi\left[\frac{-\delta-\mathrm{E}\left[D\left(t_{\mathrm{s}}, t_{\mathrm{c}}\right)\right]}{\mathrm{SD}\left[D\left(t_{\mathrm{s}}, t_{\mathrm{c}}\right)\right]}\right] \\
& =\Phi\left[\frac{-\delta-\frac{1}{\mu}\left(t_{\mathrm{c}}-t_{\mathrm{s}}\right),}{\sqrt{\frac{\sigma^{2}}{\mu^{3}}\left(t_{\mathrm{c}}+t_{\mathrm{s}}\right)}}\right] \\
& =\Phi\left[\frac{-\delta+\frac{1}{\mu}\left(t_{\mathrm{s}}-t_{\mathrm{c}}\right)}{\sqrt{\frac{\sigma^{2}}{\mu^{3}}\left(t_{\mathrm{c}}+t_{\mathrm{s}}\right)}}\right] .
\end{aligned}
$$

The parameters $\mu, \sigma$, and $\delta$ cannot be identified by fitting this equation to empirical data. This is most easily seen by multiplying the numerator and denominator of the term within the brackets of Equation 8 by $\mu$. This yields

$$
\operatorname{Pr}\left\{“ \mathrm{~S}>\mathrm{C} " \mid\left(t_{\mathrm{s}}, t_{\mathrm{c}}\right)\right\}=\Phi\left[\frac{-\mu \delta+t_{\mathrm{s}}-t_{\mathrm{c}}}{\sqrt{\frac{\sigma^{2}}{\mu}\left(t_{\mathrm{c}}+t_{\mathrm{s}}\right)}}\right],
$$

which shows that only the two parameters $\mu^{*}=\mu \cdot \delta$ and $\sigma^{*}=$ $\sigma^{2} / \mu$ can be identified for a single data set. An analogous proof applies to $\operatorname{Pr}\left\{“ \mathrm{C}>\mathrm{S}\right.$ " $\left.\mid t_{\mathrm{s}}, t_{\mathrm{c}}\right\}$ and $\operatorname{Pr}\left\{“ \mathrm{~S}=\mathrm{C} " \mid t_{\mathrm{s}}, t_{\mathrm{c}}\right\} ; \operatorname{Pr}\{“ \mathrm{~S}=$ C" $\left.\mid t_{\mathrm{s}}, t_{\mathrm{c}}\right\}$ is computed from

$$
\operatorname{Pr}\left\{“ \mathrm{~S}=\mathrm{C} " \mid t_{\mathrm{s}}, t_{\mathrm{c}}\right\}=1-\operatorname{Pr}\left\{“ \mathrm{~S}>\mathrm{C} " \mid t_{\mathrm{s}}, t_{\mathrm{c}}\right\}
$$$$
-\operatorname{Pr}\left\{“ \mathrm{C}>\mathrm{S} \text { ”| } t_{\mathrm{s}}, t_{\mathrm{c}}\right\} \text {. }
$$ 
APPENDIX B

Bounds for $\mu, \sigma$, and $\delta$

Under the reasonable assumption that the coefficient of variation-that is, $k=\sigma / \mu$, of the interpulse time distribution is less than one, as most renewal processes suggest (Cox, 1967), it is possible to establish bounds for $\delta, \mu$, and $\sigma$ and also derive a testable prediction. Thus, we can write $\sigma=k \cdot \mu$ and assume that $k \leq 1$ holds. (For a Possion process, $k$ is equal to one.)

First, consider the ratio $\mu^{*} / \sigma^{*}$, which under the assumption $\sigma=k \cdot \mu$ reduces to

$$
\frac{\mu^{*}}{\sigma^{*}}=\frac{\delta \cdot \mu}{\sigma^{2} / \mu}=\frac{\delta \cdot \mu^{2}}{\sigma^{2}}=\frac{\delta}{k^{2}} \geq 1 .
$$

Hence, this ratio should be larger than one, and in fact, our results clearly validate this prediction.

Second, from the above result one obtains an upper bound for $\delta$. Rearranging Equation 11 yields

$$
\delta=\frac{\mu^{*}}{\sigma^{*}} \cdot k^{2},
$$

and since $k^{2} \leq 1$, this establishes the upper bound

$$
\delta \leq \frac{\mu^{*}}{\sigma^{*}} .
$$

Hence, the ratio $\mu^{*} / \sigma^{*}$ defines the maximum that $\delta$ may attain.

Third, from the preceding result, we can establish a lower bound for $\mu$. Rearranging the equation $\mu^{*}=\delta \cdot \mu$ and noting that $\delta$ must be smaller than $\mu^{*} / \sigma^{*}$ yields

$$
\begin{aligned}
& \mu=\frac{\mu^{*}}{\delta} \\
& \geq=\frac{\mu^{*}}{\mu^{*} / \sigma^{*}} \\
& \geq=\sigma^{*} .
\end{aligned}
$$

Therefore, under the above assumption, $\mu$ should be larger than $\sigma^{*}$.

Finally, the assumption $k \leq 1$ provides a lower bound for $\sigma$. Note that $\sigma^{*}$ can be rewritten as

$$
\sigma^{*}=\frac{\sigma^{2}}{\mu}=k \cdot \sigma
$$

From the rightmost expression and the above assumption, it follows that $\sigma \geq \sigma^{*}$ should hold —that is, $\sigma$ must be larger than $\sigma^{*}$. 